\title{
What's new in atopic eczema? An analysis of systematic reviews published in 2015. Part 2: prevention and treatment
}

\author{
A. Lloyd-Lavery, ${ }^{1}$ N. K. Rogers, ${ }^{2}$ (D) E. Davies, ${ }^{3}$ D. J. C. Grindlay ${ }^{2}$ (D) and K. S. Thomas ${ }^{2}$ (iD \\ ${ }^{1}$ Department of Dermatology, Churchill Hospital, Oxford University Hospitals NHS Foundation Trust, Oxford, UK; ${ }^{2}$ Centre of Evidence Based Dermatology, \\ University of Nottingham, King's Meadow Campus, Lenton Lane, Nottingham, UK; and ${ }^{3}$ Department of Dermatology, The Royal Liverpool and Broadgreen \\ University Hospitals, Thomas Drive, Liverpool, UK
}

doi:10.1111/ced. 13554

\section{Summary}

\begin{abstract}
This review forms part of a series of annual updates that summarize the evidence base for atopic eczema (AE), providing a succinct guide for clinicians and patients. It provides a summary of key findings from 26 systematic reviews that were published during 2015 , and focuses on the treatment and prevention of AE. For systematic reviews on the epidemiology and methodological issues, see Part 1 of this update. Topical corticosteroid withdrawal syndrome, 'steroid addiction', has been evaluated in a high-quality systematic review, which helps better define this entity and the risk factors for it. A Cochrane Review has not demonstrated any association between topical corticosteroid use in pregnancy and adverse outcomes, although very large quantities of potent/very potent topical corticosteroids may be associated with reduced birth weight. House dust mite avoidance strategies do not appear to prevent AE. Exposure to probiotics prenatally and in early infancy may help prevent $\mathrm{AE}$, but there is no evidence that maternal diet or supplementation has a preventative effect.
\end{abstract}

\section{Background}

Atopic eczema (AE) is one of the most prevalent diseases worldwide, ${ }^{1}$ and its study is a fast-moving field. The aim of this evidence update is to highlight the clinical and research implications from systematic reviews (SRs) published during 2015, summarizing the findings of these SRs on the treatment and prevention of AE. For an update of SRs on the methodological issues and epidemiology of AE, see Part 1 of this update. Similar evidence updates on $\mathrm{AE}$ have been published previously, along with the details of the search methods used. ${ }^{2-4}$

Correspondence: Professor Kim S. Thomas, Centre of Evidence Based Dermatology, University of Nottingham, King's Meadow Campus, Lenton Lane, Nottingham, NG7 2NR, UK

E-mail:kim.thomas@nottingham.ac.uk

Conflict of interest: none declared.

Accepted for publication 8 August 2017

\section{Treatment of atopic eczema}

\section{Emollients}

Emollients are a mainstay of $\mathrm{AE}$ treatment, but are poorly investigated. The review by Lindh and Bradley ${ }^{5}$ of moisturizer efficacy evaluated $32 \mathrm{AE}$ studies, including 25 randomized controlled trials (RCTs), with a total of 2332 participants. Most studies reported beneficial effects on clinical symptoms, transepidermal water loss (TEWL) and stratum corneum (SC) hydration, with the clinical effect appearing greatest for products containing urea. Studies were mostly of short duration (ranging from 90 min to 6 months), involved few patients (range 10-173) and carried a high risk of bias. Meta-analysis was precluded owing to significant heterogeneity in the control intervention and the measured outcomes.

\section{Topical corticosteroids: side effects and safety}

Three SRs assessed various safety considerations surrounding topical corticosteroid (TCS) use. 
TCS use and skin atrophy was evaluated in a review covering 15 AE studies (8 RCTs, 7 open-label observational studies). ${ }^{6}$ The authors reported that the benefit/risk ratio depended on TCS potency. Study duration ranged from 2 to 52 weeks, but no specific data were included on the duration of any local effects post-TCS cessation. Whether subclinical atrophy translates to clinically meaningful changes is still unclear.

Hajar et al. ${ }^{7}$ conducted an SR assessing TCS withdrawal syndrome ('steroid addiction') an adverse effect arising from TCS withdrawal after prolonged or inappropriate use of moderate- to high-potency TCS, mainly on the face and, to a lesser extent, the genitals. The review assessed 34 studies (19 case series, 13 case reports, 2 cross-sectional studies), involving 251 patients with AE, most of whom were female. Erythema was noted in $92 \%$ of patients, with papulonodular or pustular lesions present in approximately $50 \%$. No effective management strategy was established, although TCS discontinuation was recommended.

An updated Cochrane Review ${ }^{8}$ evaluated the effect of TCS use on pregnancy outcomes, with a total of 1601515 patients. Most studies found no causal associations between maternal exposure to TCS and adverse pregnancy outcomes. However, a probable association between low birth weight and sustained maternal use of potent to very potent TCS was reported. The overall quality of the evidence was low.

\section{Topical calcineurin inhibitors}

A Cochrane Review ${ }^{9}$ assessed the efficacy and safety of topical tacrolimus for moderate to severe AE, with a total of 5885 patients in 20 RCTs, which were mostly short-term (range 13 days to 6 months). Tacrolimus $(0.03 \% ; 0.1 \%)$ was more effective than mild-potency TCS and pimecrolimus, but results were equivocal compared with moderate to potent TCS. Transient itching and burning were more commonly observed with tacrolimus than with TCS, but there was no difference in the rate of skin infections. There was no evidence to support an increased risk of malignancy with the use of tacrolimus. Variation in drug dose, outcomes and follow-up periods precluded meta-analysis. A separate review of SRs assessing topical calcineurin inhibitors in $\mathrm{AE}^{10}$ concluded that tacrolimus is an effective and cost-effective alternative treatment to TCS. No formal quality assessment of the included studies was performed.

\section{Phototherapy}

Perez-Ferriols ${ }^{11}$ and colleagues evaluated the efficacy of photo(chemo)therapy for moderate to severe AE. Evidence from 21 studies with a total of 961 patients supported narrowband ultraviolet B (NB-UVB) and UVA1 phototherapy use, but data on long-term effects, particularly in childhood, were limited. Few data were available regarding the efficacy of psoralen (P)UVA, and further studies using conventional treatment schedules are needed to establish the relative efficacy of NB-UVB and UVA1. An SR by Dogra et al. ${ }^{12}$ drew similar conclusions.

\section{Psychological and educational interventions and approaches to improving atopic eczema treatment}

There have been three SRs of psychological and educational interventions for AE published in the period of this update. Eccleson and colleagues ${ }^{13}$ updated their Cochrane Review on psychological therapies for parents of young people with chronic illness. Only one study related to $\mathrm{AE}$ was included, and this compared parental education using behavioural-based group education or video education with standard care. The authors suggested that parental education, particularly video education, improved AE symptoms overall, but there was a high risk of both attrition and reporting bias, making it difficult to draw firm conclusions.

A health technology assessment ${ }^{14}$ of educational interventions to improve health-related quality of life (QoL) included two RCTs involving children/adolescents with $\mathrm{AE}$ and their carers (1141 participants) and an RCT of 50 adult patients with either AE or psoriasis. Despite some evidence hinting at possible benefit, there is uncertainty as to whether educational interventions are clinically effective or cost-effective, and which types of interventions are most likely to be beneficial in different countries. Lee et al. ${ }^{15}$ qualitatively assessed 15 studies of educational programmes for children with $\mathrm{AE}$, and proposed four key themes to be included in $\mathrm{AE}$ support interventions: (i) tailored support by age or $\mathrm{AE}$ severity, with a patient- and family-centred approach; (i) well-trained educators (evidence particularly favouring nurse-led programmes and topical demonstrations); (iii) use of diverse educational methods including webbased education; and (iv) focus on emotional support and improving QoL of children and their families.

A review of nine studies (including four RCTs) ${ }^{16}$ investigated multidisciplinary approaches for patients (encompassing educational, psychological, behavioural and nutritional interventions), and found that most studies demonstrated improvements in AE severity. 
There were considerable limitations, including variability in study design and outcomes.

Bass et al. ${ }^{17}$ evaluated different methods to increase adherence to $\mathrm{AE}$ treatment (indirectly inferred from changes in $\mathrm{AE}$ severity or quality of life in many cases). Assessing the 7 included studies (760 participants), the authors concluded that written eczema action plans, extra clinic visits and educational workshop attendance may improve treatment adherence. They advised further research on how currently available treatments could be better utilized.

In summary, despite some suggestion of benefit, it is not clear which types of psychological/educational interventions work best, and how these may need to be tailored depending on the resource limitations and patient needs in different countries.

\section{Acupuncture}

Two SRs investigated acupuncture efficacy in AE. Tan et al., ${ }^{18}$ who strictly defined $\mathrm{AE}$ for inclusion in the review, found no RCTs. By contrast, Ma and colleagues ${ }^{19}$ reported 5 RCTs (four by the same research group), and 1 prospective observational study, which had a total of 170 participants. Although itch measurements were lower in the acupuncture groups, the studies were small, and the acupuncture regimens and control arms varied significantly.

\section{Bathing}

Sarre $e t a .^{20}$ evaluated the impact of water submersion (5-15 min) on the skin of patients with $\mathrm{AE}$ from 4 heterogeneous studies ( 3 RCTs, 1 crossover) with 63 participants in total. The review concluded that bathing had no impact on various functional parameters, including TEWL and SC hydration. However, the included studies were small and heterogeneous, while important potential confounders, such as concomitant use of emollients or water temperature, were not addressed.

\section{House dust mite avoidance for treatment of atopic eczema}

Nankervis et al. ${ }^{21}$ performed a Cochrane Review assessing house dust mite reduction and avoidance measures for the treatment of $\mathrm{AE}$, which assessed 7 RCTs with a total of 324 participants. Modest treatment responses with dust mite reduction techniques were reported in one small, low-quality study of patients with $\mathrm{AE}$ with aeroallergen sensitivities, but the value and optimal timing of house dust mite reduction for patients with $\mathrm{AE}$ in general is unknown. Overall, the evidence was of very low quality.

\section{Prevention of atopic eczema}

\section{Probiotics}

Zuccoti et al. ${ }^{22}$ evaluated 17 heterogeneous RCTs with a total of 4755 parents and children. Prenatal and perinatal exposure to probiotics (in either the mother or child after birth) decreased the risk of AE development in children at high risk of atopy (risk ratio 0.78; $95 \%$ CI $0.69-0.89 ; \quad P=0.0003)$, with a number needed to treat of 13. The preventative effect appeared confined to the first 24 months of life. The observed effects were most convincing when probiotic mixtures (rather than single strains) were used. Two other SRs gave similar findings, although one found probiotics were associated with a reduced $\mathrm{AE}$ risk over a sustained period ( $>5$ years) ${ }^{23,24}$ It is unclear which probiotic strains are most effective, or when these are best delivered during pregnancy and early life.

\section{Maternal diet}

A review by Beckhaus et al. ${ }^{25}$ of 24 studies (23 prospective, 1 retrospective) found insufficient evidence to recommend any maternal diet or supplementation to prevent childhood $\mathrm{AE}^{25}$

A Cochrane Review ${ }^{26}$ of six RCTs found that prenatal $n-3$ long-chain polyunsaturated fatty acid (LCPUFA) dietary supplementation did not reduce $\mathrm{AE}$ incidence. A decrease in IgE-mediated AE between 1 and 3 years of age was reported in two trials including high-risk patients. Studies included different regimens, making direct comparisons difficult.

Another review by Best et al. ${ }^{27}$ evaluating prenatal $n$ 3 LCPUFA exposure for AE prevention in eight prospective cohort studies and four RCTs found inconsistent evidence. A meta-analysis demonstrated a significant reduction in $\mathrm{AE}$ incidence (defined as eczema symptoms and a positive skin prick test) at 12 months (relative risk 0.53 ; 95\% CI $0.35-0.81 ; P=0.004$ ), but this benefit was lost by 3 years of age. There was significant variation in diet and regimen timings between studies, making meaningful conclusions difficult to draw.

\section{Breast feeding}

Lodge et al. ${ }^{28}$ reviewed 42 observational studies (with 472488 participants) and found low- to very low- 
quality evidence that breastfeeding reduces the risk of eczema development up to 2 years of age. After this age, the protective effect was lost. The studies failed to adjust for important confounders, including a family history of atopy, and there were often long recall periods.

\section{House dust mite avoidance}

A review by Bremmer et al. ${ }^{29}$ of 7 RCTs with 3040 patients indicated that dust mite avoidance strategies, alone or in combination with other allergen avoidance strategies, did not reduce the risk of developing AE. The included studies mostly had a moderate risk of bias, and the criteria used to diagnose AE were unclear. Combining three trials using a dust mite avoidance approach in a meta-analysis showed no benefit in the primary prevention of $\mathrm{AE}(\mathrm{RR}=1.08 ; 95 \% \mathrm{CI}$ 0.78-1.49).

\section{Learning points}

- Although emollients are a mainstay treatment for $\mathrm{AE}$, they have been poorly investigated to date, and head-to-head comparisons are lacking. - TCS withdrawal syndrome ('steroid addiction') appears to be a distinct adverse effect of TCS use, resulting from prolonged, inappropriate or frequent use of moderate- to high-potency TCS, particularly on the face.

- The use of TCS during pregnancy does not appear to be related to adverse pregnancy outcomes, although the use of very large quantities of potent to very potent TCS may be associated with low birth weight.

- Evidence supports the use of narrowband UVB and UVA1 phototherapy to treat AE, but data for treating childhood AE with phototherapy are limited and there are few data on long-term effects.

- Very low-quality evidence indicates that house dust mite reduction and avoidance measures may be beneficial for patients with $\mathrm{AE}$ who have aeroallergen sensitivities, but these approaches do not appear to prevent the development of AE.

- Exposure to probiotics during pregnancy and in early infancy may reduce the risk of developing $\mathrm{AE}$, although the duration of this protective effect is unclear.

- There is no consistent evidence that maternal diet helps to prevent AE, but there is some evidence that $n-3$ long chain polyunsaturated fatty acid dietary supplementation may reduce the incidence of $\mathrm{AE}$.

\section{Acknowledgement}

This work was carried out as part of the UK Dermatology Clinical Trials Network (UK DCTN) Fellowship scheme.

\section{References}

1 Hay RJ, Johns NE, Williams HC et al. The global burden of skin disease in 2010: an analysis of the prevalence and impact of skin conditions. J Invest Dermatol 2014; 134: 1527-34.

2 Hatfield SJ, Rogers NK, Lloyd-Lavery A et al. What's new in atopic eczema? An analysis of systematic reviews published in 2014. Part 1. Epidemiology, risk factors and outcomes. Clin Exp Dermatol 2016; 41: 843-6.

3 Lloyd-Lavery A, Rogers NK, Hatfield SJ et al. What's new in atopic eczema? An analysis of systematic reviews published in 2014. Part 1. Epidemiology, risk factors and outcomes. Clin Exp Dermatol 2017; 42: 3-7.

4 Centre of Evidence Based Dermatology. Annual Evidence Updates for Eczema - Guidance. Available at: https:// www.nottingham.ac.uk/research/groups/cebd/documents/ methodological-resources/ebu-protocol.pdf (accessed 1 April 2017)

5 Lindh JD, Bradley M. Clinical effectiveness of moisturizers in atopic dermatitis and related disorders: a systematic review. Am J Clin Dermatol 2015; 16: 341-59.

6 Barnes L, Kaya G, Rollason V. Topical corticosteroidinduced skin atrophy: a comprehensive review. Drug Saf 2015; 38: 493-509.

7 Hajar T, Leshem YA, Hanifin JM et al. A systematic review of topical corticosteroid withdrawal ("steroid addiction") in patients with atopic dermatitis and other dermatoses. J Am Acad Dermatol 2015; 72: 541-9.e2.

8 Chi CC, Wang SH, Wojnarowska F et al. Safety of topical corticosteroids in pregnancy. Cochrane Database Syst Rev 2015; (10): CD007346.

9 Cury Martins J, Martins C, Aoki V et al. Topical tacrolimus for atopic dermatitis. Cochrane Database Syst Rev 2015; (7): CD009864.

10 Chia BK, Tey HL. Systematic review on the efficacy, safety, and cost-effectiveness of topical calcineurin inhibitors in atopic dermatitis. Dermatitis 2015; 26: 122-32.

11 Perez-Ferriols A, Aranegui B, Pujol-Montcusi JA et al. Phototherapy in atopic dermatitis: a systematic review of the literature. Actas Dermosifiliogr 2015; 106: 387-401.

12 Dogra S, Mahajan R. Indian Association of Dermatologists Venereologists Leprologists. Phototherapy for atopic dermatitis. Indian J Dermatol Venereol Leprol 2015; 81: 10-15. 
13 Eccleston C, Fisher E, Law E et al. Psychological interventions for parents of children and adolescents with chronic illness. Cochrane Database Syst Rev 2015; (4): CD009660.

14 Pickett K, Loveman E, Kalita N et al. Educational interventions to improve quality of life in people with chronic inflammatory skin diseases: systematic reviews of clinical effectiveness and cost-effectiveness. Health Technol Assess 2015; 19: 1-176, v-vi.

15 Lee Y, Oh J. Educational programs for the management of childhood atopic dermatitis: an integrative review. Asian Nurs Res 2015; 9: 185-93.

16 Spielman SC, LeBovidge JS, Timmons KG et al. A review of multidisciplinary interventions in atopic dermatitis. $J$ Clin Med 2015; 4: 1156-70.

17 Bass AM, Anderson KL, Feldman SR. Interventions to increase treatment adherence in pediatric atopic dermatitis: a systematic review. J Clin Med 2015; 4: 231-42.

18 Tan HY, Lenon GB, Zhang AL et al. Efficacy of acupuncture in the management of atopic dermatitis: a systematic review. Clin Exp Dermatol 2015; 40: 711-15; quiz 5-6.

19 Ratib S, Wilkes SR, Nankervis H et al. How often do comparative randomised controlled trials in the field of eczema fail to directly compare the treatments being tested? J Clin Med 2015; 4: 1312-24.

20 Sarre ME, Martin L, Moote W et al. Are baths desirable in atopic dermatitis? J Eur Acad Dermatol Venereol 2015; 29: 1265-74.

21 Nankervis H, Pynn EV, Boyle RJ et al. House dust mite reduction and avoidance measures for treating eczema. Cochrane Database Syst Rev 2015; (1): CD008426.

22 Zuccotti G, Meneghin F, Aceti A et al. Probiotics for prevention of atopic diseases in infants:

\section{CPD questions}

\section{Learning objective}

To demonstrate up-to-date knowledge of prevention and treatment in atopic eczema.

\section{Question 1}

Which of the following statements regarding topical treatments for atopic eczema (AE) is true?

(a) There is evidence indicating a probable association between the use of potent to very potent topical corticosteroids in pregnancy and craniofacial congenital abnormalities.

(b) A Cochrane Review found evidence for an increased risk of skin infections with tacrolimus use compared with topical corticosteroids.

(c) Mild-potency topical corticosteroids were found to be equivalent in efficacy to tacrolimus $0.1 \%$. systematic review and meta-analysis. Allergy 2015; 70: 1356-71.

23 Cao L, Wang L, Yang L et al. Long-term effect of earlylife supplementation with probiotics on preventing atopic dermatitis: a meta-analysis. J Dermatolog Treat 2015; 26: 537-40.

24 Cuello-Garcia CA, Brozek JL, Fiocchi A et al. Probiotics for the prevention of allergy: a systematic review and meta-analysis of randomized controlled trials. J Allergy Clin Immunol 2015; 136: 952-61.

25 Beckhaus AA, Garcia-Marcos L, Forno E et al. Maternal nutrition during pregnancy and risk of asthma, wheeze, and atopic diseases during childhood: a systematic review and meta-analysis. Allergy 2015; 70: 1588-604.

26 Gunaratne AW, Makrides M, Collins CT. Maternal prenatal and/or postnatal n-3 long chain polyunsaturated fatty acids (LCPUFA) supplementation for preventing allergies in early childhood. Cochrane Database Syst Rev 2015; (7): CD010085.

27 Best KP, Gold M, Kennedy D et al. Omega-3 long-chain PUFA intake during pregnancy and allergic disease outcomes in the offspring: a systematic review and metaanalysis of observational studies and randomized controlled trials. Am J Clin Nutr 2016; 103: 128-43.

28 Lodge CJ, Tan DJ, Lau MX et al. Breastfeeding and asthma and allergies: a systematic review and metaanalysis. Acta Paediatr 2015; 104: 38-53.

29 Bremmer SF, Simpson EL. Dust mite avoidance for the primary prevention of atopic dermatitis: a systematic review and meta-analysis. Pediatr Allergy Immunol 2015; 26: 646-54.

(d) Evidence suggests that the beneficial clinical effects of moisturizers may be highest for those containing urea.

(e) A Cochrane Review found evidence for an increased risk of malignancy with tacrolimus use.

\section{Question 2}

Which of the following statements regarding topical corticosteroid (TCS) 'addiction'/withdrawal is correct?

(a) This is most commonly seen in male patients.

(b) It can be the consequence of a short period of a very potent TCS on the face.

(c) The genitals are the most commonly affected site.

(d) Erythema is the most common sign.

(e) The topical corticosteroid should be continued. 


\section{Question 3}

Which of the following statements regarding the prevention of $\mathrm{AE}$ is true?

(a) Maternal intake of $n-3$ long chain polyunsaturated fatty acids may reduce the incidence of $\mathrm{AE}$.

(b) Maternal intake of probiotics during pregnancy does not appear to reduce the incidence of $\mathrm{AE}$ in the first 24 months of life.

(c) Breastfeeding may reduce the long-term incidence of $\mathrm{AE}$.

(d) House dust mite avoidance strategies may reduce the risk of developing AE.

(e) Maternal intake of vitamin D may reduce the incidence of $\mathrm{AE}$.

\section{Question 4}

Recent systematic reviews have found robust evidence of effectiveness for which treatment for AE?

(a) Psoralen ultraviolet A (PUVA) phototherapy.

(b) Acupuncture.

(c) Emollients.

(d) Educational programmes.

(e) Topical tacrolimus.

\section{Question 5}

Which of the following statements about treatments for $\mathrm{AE}$ is true?

(a) Short-term tap-water submersion of AE skin has been shown to increase transepidermal water loss. (b) Multidisciplinary approaches to management have not been shown to affect AE severity.

(c) Evidence suggests that patients with AE should use house dust mite reduction and avoidance measures.

(d) Written eczema action plans have been associated with improved AE treatment adherence.

(e) A number of studies indicate that psychological therapies are helpful for managing AE.

\section{Instructions for answering questions}

This learning activity is freely available online at http://www.wileyhealthlearning.com/ced

Users are encouraged to

- Read the article in print or online, paying particular attention to the learning points and any author conflict of interest disclosures

- Reflect on the article

- Register or login online at http://www.wileyhealth learning.com/ced and answer the CPD questions

- Complete the required evaluation component of the activity

Once the test is passed, you will receive a certificate and the learning activity can be added to your RCP CPD diary as a self-certified entry.

This activity will be available for CPD credit for 2 years following its publication date. At that time, it will be reviewed and potentially updated and extended for an additional period. 